\title{
FM 047-02: a collisional pair of galaxies with a ring ${ }^{\star}$
}

\author{
M. Faúndez-Abans ${ }^{1}$, M. de Oliveira-Abans ${ }^{1,2}$, A. C. Krabbe $^{3}$ \\ P. C. da Rocha-Poppe ${ }^{4,5}$, V. A. Fernandes-Martin ${ }^{4,5}$, and I. F. Fernandes ${ }^{4,5}$
}

\author{
${ }^{1}$ MCTI/Laboratório Nacional de Astrofísica, Rua Estados Unidos, 154, Bairro das Nações, CEP 37.504-364 Itajubá, MG, Brazil \\ e-mail: [mfaundez;mabans]@lna.br \\ 2 UNIFEI, Instituto de Engenharia de Produção e Gestão, Av. BPS 1303 Pinheirinho, 37500-903 Itajubá, MG, Brazil \\ 3 Universidade do Vale do Paraíba, UNIVAP. Av. Shishima Hifumi, 2911, Urbanova CEP: 12244-000, São José dos Campos, SP, \\ Brazil \\ e-mail: angela.krabbe@gmail.com \\ 4 UEFS, Departamento de Física, Av. Transnordestina, S/N, Novo Horizonte, CEP 44036-900 Feira de Santana, BA, Brazil \\ 5 UEFS, Observatório Astronômico Antares, Rua da Barra, 925, Jardim Cruzeiro, CEP 44024-432 Feira de Santana, BA, Brazil \\ e-mail: [paulopoppe; vmartin1963;irafbear]@gmail.com
}

Received 22 January 2013 / Accepted 17 July 2013

\section{ABSTRACT}

\begin{abstract}
Aims. We investigate the nature of the galaxy pair FM 047-02, which has been proposed as an archetype of the Solitaire types of peculiar (collisional) ring galaxies.

Methods. The study is based on long-slit spectrophotometric data in the range of 3500-9500 A obtained with the Gemini Multi-Object Spectrograph at Gemini South (GMOS-S). The absorption spectra were used to determine the radial velocity. The stellar population synthesis code STARLIGHT was used to investigate the star formation history of the galaxy pair FM 047-02.

Results. During the whole long-slit signal, the spectra of both galaxies resemble those of an early-type galaxy. Both objects are dominated by an old stellar population of $2 \times 10^{9}<t \leq 13 \times 10^{9} \mathrm{yr}$ and a small, but a non-negligible fraction (about $13 \%$ ) of young stars of $t \leq 5 \times 10^{7} \mathrm{yr}$ are estimated to contribute to the optical flux for NED02. Both observed small radial-velocity differences, and the structures around NED01 suggest an ongoing tidal interaction of both galaxies.

Conclusions. The spectroscopic results and the morphological peculiarities of NED01 can be adequately interpreted as continuing off-center stage of interaction with the companion galaxy NED02. The core of NED01 and NED02 are in counter-rotating motion. The visual appearance of NED01, its kinematical properties, the smooth distribution of material in the ring, and its off-centered nucleus, characterize it as a true archetype of a Solitaire-type ring galaxy in an advanced stage of ring formation.
\end{abstract}

Key words. galaxies: general - galaxies: peculiar - galaxies: individual: FM047-02 - galaxies: interactions

\section{Introduction}

Today it is well accepted that most galaxies experience several collisions and/or tidal interactions over the course of their lifetimes. Some of these interactions may be strong enough to profoundly alter their structure and accelerate their evolutionary process. Thus, collisions and interactions are now generally believed to be one of the primary drivers of galaxy evolution and morphological transformation of the structure of a galaxy. The processes of galaxy formation and evolution are intimately connected to star formation, as are a variety of other characteristics related to galaxies, such as the creation of heavy elements in the Universe by stellar evolution and the formation of galactic structures, planetary systems, production and distribution of cosmic rays, among others. One of the possible results of collisions and tidal interactions in galaxies are rings triggered in galactic disks.

It is noteworthy here to clarify that not all ring galaxies have the same origin. They can be divided into two main groups: the normal Ring galaxies (NRG) and the peculiar Ring galaxies (pRG) (Faúndez-Abans \& de Oliveira-Abans 1998, hereafter FAOA). The rings of NRG result from resonances between the

* Based on observations made at the Gemini Observatory, under the identification number GS-2007A-DD-06. gravitational field of structures, such as bars, ovals, or spiral arms, and their oscillation about the circular orbits in a galactic disk. The pRGs are the result of tidal interaction between two galaxies, a collisional scenario that ends in the merger and transmutation of the objects (Appleton \& Struck-Marcell 1996). The pRGs show a wide variety of ring and bulge morphologies and are classified by Faúndez-Abans \& de OliveiraAbans (1998) into five families, following the general behavior of galaxy-ring structures. Eight morphological subdivisions are highlighted for these categories. One of them is a basic structure called "Solitaire". The Solitaire pRG is described there as an object with the bulge on the ring or very close to it, resembling a one-diamond finger ring (single knotted ring). In these objects, the ring generally looks smooth and almost thin on the opposite side of the bulge (for archetypes see FM 18815/NED02, AM 0436-472/NED01, ESO 202-IG45/NED01, and ESO 303-IG11/NED01). There are still a few statistical works on this type of object, and details of the collisional process behind their formation is still unknown.

In this work, we report a first study with spectroscopic observations of the tidally disturbed galaxy NED01, a member of the galaxy pair FM 047-02 (ESO 046-IG10; AM 2021-724; see also Arp \& Madore 1986) and its companion NED02, based on long-slit spectrophotometric data 
Table 1. Data on FM 047-02 galaxies.

\begin{tabular}{lccc}
\hline \hline Parameter & NED01 & NED02 & Ref. \\
\hline RA $(2000)$ & 202629.5 & 202633.9 & NED \\
Dec $(2000)$ & -723644.5 & -723608.2 & NED \\
Morphology & Solitaire & E4: & this work \\
pRG-family & Solitaire-like & & FAOA \\
$z$ & 0.0767 & 0.0759 & this work \\
$V\left(\mathrm{~km} \mathrm{~s}^{-1}\right)^{*}$ & $22994 \pm 25$ & $22754 \pm 20$ & this work \\
Distance $(\mathrm{Mpc})$ & 322 & 322 & this work \\
$\sigma_{v}\left(\mathrm{~km} \mathrm{~s}^{-1}\right)$ & 387 & 285 & this work \\
Mass (lower limit) & $3.97 \times 10^{11} M_{\odot}$ & $1.93 \times 10^{11} M_{\odot}$ & this work \\
$R_{\text {eff }}($ adopted $)$ & $12^{\prime \prime} 7$ & $11^{\prime \prime} 5$ & this work \\
\hline
\end{tabular}

Notes. ${ }^{(*)}$ Non relativistic velocity using the standard formula (Lang 1999).

obtained with the Gemini GMOS Spectrograph at the Gemini Observatory (ID: GS-2007A-DD-06) in Chile. A value of $H_{\mathrm{o}}=$ $70 \mathrm{~km} \mathrm{~s}^{-1} \mathrm{Mpc}^{-1}, \Omega_{\text {matter }}=0.27$ and $\Omega_{\text {vaccum }}=0.73$ have been adopted throughout this work (Freedman et al. 2001; Astier et al. 2006; see also Spergel et al. 2003). Table 1 presents this work's new results for both galaxies.

\section{Observations and data reduction}

The FM 047-02 galaxy pair is composed of a ringed galaxy, NED01 (2MASX J20262950-7236443); and an elliptical-like object, NED02 (2MASX J20263396-7236083). Figure 1 displays both galaxies from the telescope pointing 5 min-exposure GMOS image ( $r$-G0303 filter with an effective wavelength of $630 \mathrm{~nm}$ ). The spectroscopic observations were performed with the 8.1-m Gemini South telescope, Chile (ID program GS-2007A-DD-06). We used the GMOS spectrograph in long-slit mode (Hook et al. 2004) ${ }^{1}$. The long slit used has an entrance on the plane of the sky of $1.5^{\prime \prime} \times 375^{\prime \prime}$. A grating of 400 lines/mm (R400+G5325, with GG455-G0329 blocking filter) centered on $676.5 \mathrm{~nm}$ was used. The data were binned by two pixels in both the spatial and spectral dimensions, producing a spectral resolution of $\sim 5.1 \AA F W H M$ sampled at $0.68 \AA \mathrm{pix}^{-1}$. The seeing throughout the observations was $1^{\prime \prime} .5$. The binned pixel scale was 0 .' $^{\prime} 145 \mathrm{pix}^{-1}$. The wavelength range is 3500-9500 $\AA$. The star LTT 7379 was used for extinction and flux calibrations. It is a tertiary standard from Baldwin \& Stone (1984), as revised by Hamuy et al. (1992, see also Hamuy et al. 1994). The long-slit spectra were taken at one position angle on the sky, $\mathrm{PA}=28^{\circ}$, to cover both objects in one shot and across the center of both objects.

The standard Gemini-IRAF routines were used to carry out bias subtraction, flat-fielding, and cosmic ray hits. The data were then calibrated in wavelength with an accuracy of $\leqslant 0.3 \AA$. The 2D spectra was then extracted into 1D spectra which were sky-subtracted and binned in the spatial dimension. The binned 1D spectra were flux-calibrated using the spectrophotometric standard star LTT 7379.

\footnotetext{
1 A description of the instrument can be found at http://www . gemini . edu/sciops/instruments/gmos
}

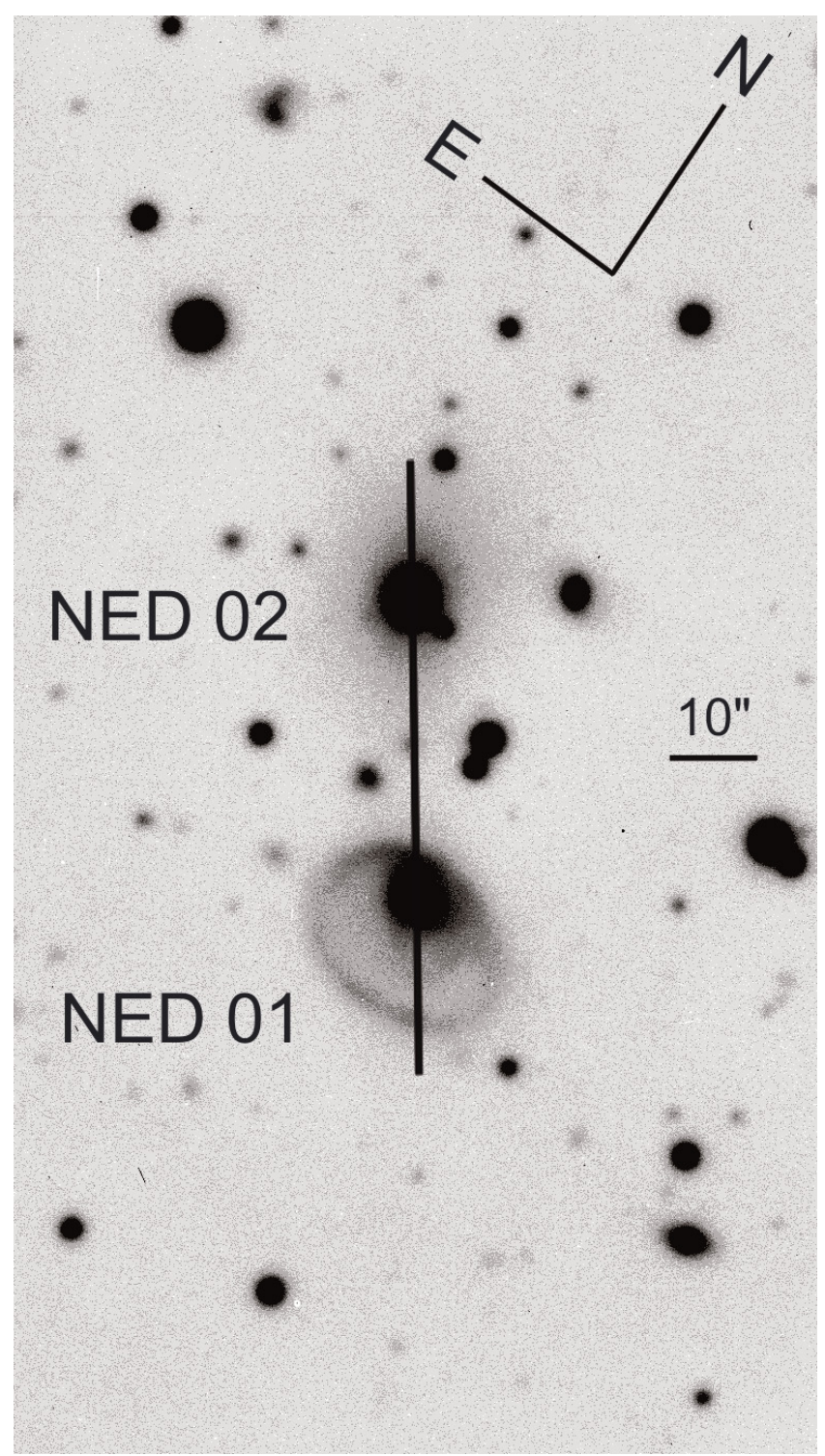

Fig. 1. Solitaire galaxy NED01 and its companion NED02. Original optical GMOS pointing image in the $r$-G0303 filter, with contrast to highlight the main morphological features of the galaxies. The PA $=28^{\circ}$ slit position is displayed.

\section{Kinematics}

The radial velocity for each galaxy was estimated by the cross-correlation technique. Using IRAF/RVXCOR we cross-correlated our observed spectra with three galaxy and star templates with high signal-to-noise. These results were checked with the composite absorption-line template "fabtemp97" distributed by the RVSAO ${ }^{2}$ /IRAF external package. We adopted the redshift value from the best high correlated coefficient template. Figures 2 and 3 display the distribution of the radial velocity for NED01 and NED02, respectively. Figure 4 presents a sample of integrated spectra in rest frame and the model stellar population espectrum in the range of 4600-6500 $\AA$ for NED01 and NED02, respectively (see Sect. 4 for more details). The spectra

2 The RVSAO IRAF (Radial Velocity Package for IRAF) external package was developed at the Smithsonian Astrophysical Observatory. Full documentation of this software, including numerous examples of its use, is online at http://tdc-www.harvard.edu/iraf/rvsao/ 

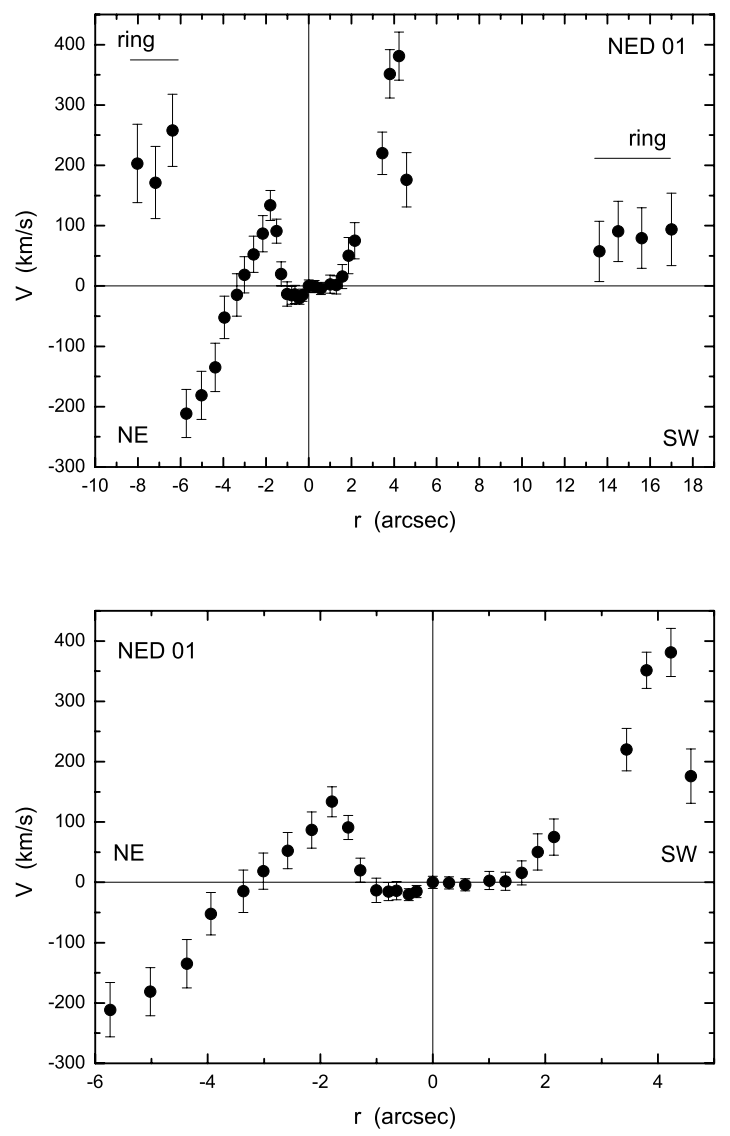

Fig. 2. From top to bottom: a) the NED01 rotation profile of the nucleus, bulge and ring sections, the total long-slit $-10^{\prime \prime}$ to $+19^{\prime \prime}$ distribution (which shows a U-shaped rotation profile at the center); b) the central $-6^{\prime \prime}$ to $+5^{\prime \prime}$ nuclear and bulge features.

had significant fringing in the red (7000-9500 $\mathrm{A})$, and no emission lines in both spectra were detected along the entire slit, even in the selected regions of the NED01 ring. This region of the spectra with significant fringing was not used in our analysis.

\subsection{NED01}

The spectral profile resembles that of an early-type object. The velocity found is $V=22994 \mathrm{~km} \mathrm{~s}^{-1}(z=0.0767)$. Figure 2 displays NED01's distribution of radial velocities along the whole object (upper panel) and the central region (lower panels). The errors of the individual velocity measurements do not exceed $10 \mathrm{~km} \mathrm{~s}^{-1}$ in the central region and increase to $20-40 \mathrm{~km} \mathrm{~s}^{-1}$ on its periphery. The two off-centered main ring sections $\left(-6^{\prime \prime}\right.$ to $-8.5^{\prime \prime}$ and $13^{\prime \prime}$ to $17.5^{\prime \prime}$, respectively) and the loop-ring (close to $+4^{\prime \prime}$ north section) are receding from us.

Clearly NED01 is a tidally perturbed object. A U-shaped profile is displayed in the $\pm 4^{\prime \prime}$ distribution section of the radial velocities. This shape appears on interacting binary-disturbed elliptical galaxies (see Borne 1990; Borne \& Hoessel 1985, 1988; Bender et al. 1991; Madejsky 1991; Madejsky et al. 1991). The physical interpretation of Borne et al. (1994) is that there is a tidal coupling between the orbit of the companion and the resonant prograde rotating stars in the kinematically disturbed galaxy (Borne 1988; Borne \& Hoessel 1988; Bacells et al. 1989). This U-shape profile in NED01 is thus direct observational evidence of tidal coupling, hence a direct observational signature of tidal friction in action in this pair of galaxies.

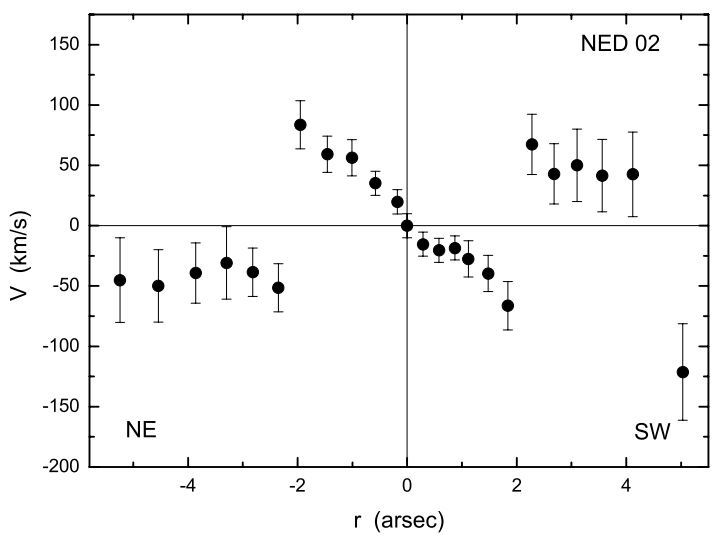

Fig. 3. NED02 observed distribution of the rotation profile of the nuclear, bulge, and external features, respectively.

Inspecting the upper panel of Fig. 2, we realize that there are several kinematical subsystems throughout the regions comprised by the slit: (1) the U-shaped base of the rotation profile; (2) the wings of the U-shaped rotation profile; (3) a rigid rotator (from $-6^{\prime \prime}$ to $-2^{\prime \prime} \mathrm{NE}-$ section; although internally wavy, the one on the rotator's left is approaching, while the one on its right is receding from us); and (4) the ring sections. The lower panel of Fig. 2 shows the observed radial velocity from $-4.5^{\prime \prime}$ to $4.5^{\prime \prime}$, and the enlarged U-shape.

\subsection{NED02}

The spectrum of NED02 shows characteristics of an early-type galaxy, which are typical of elliptical galaxies, like the dwarf ellipticals that populate the Virgo cluster of galaxies (van Zee et al. 2004). The velocity that we found is $V=22754 \mathrm{~km} \mathrm{~s}^{-1}$ $(z=0.0759)$, which is in good agreement with the previously reported value $z=0.0756$ on NED.

Figure 3 displays the distribution of radial velocities of NED02 measured along all the object. The errors of the individual velocity measurements do not exceed $10 \mathrm{~km} \mathrm{~s}^{-1}$ in the central region and increase to $20-30 \mathrm{~km} \mathrm{~s}^{-1}$ on its periphery. In this figure, the rotation curve comprising $\pm 2^{\prime \prime}$ around the nucleus is similar to those of galaxies with kinematically decoupled regions. (Balcells \& González 1998; see also Hau \& Thomson 1994; De Rijcke et al. 2004). Inspecting Fig. 3, we realize that there are two main kinematical subsystems along the observed slit: (1) a core-bulge rigid rotator at $\pm 2^{\prime \prime}$, with almost the same angular velocity as suggested by the average inclination of their linear profile (its SW part is approaching while its NE part is receding from us); and (2) the main body with a flat rotation curve around $\pm 50 \mathrm{~km} \mathrm{~s}^{-1}\left(r> \pm 2^{\prime \prime}\right)$ decoupled from the core-bulge section. The NE-section is approaching while the $\mathrm{SW}$-section is receding from us. The latter is the "contact region" between NED02 and NED01. Adopting the $b / a=0.84$ ratio from the pointing image, the morphology of NED02 suggests that it is an elliptical galaxy, maybe an E4.

\section{Stellar population synthesis}

Detailed study of the star formation in tidally perturbed galaxies provides important information, not only on the age distribution along their stellar population components, but also on several 

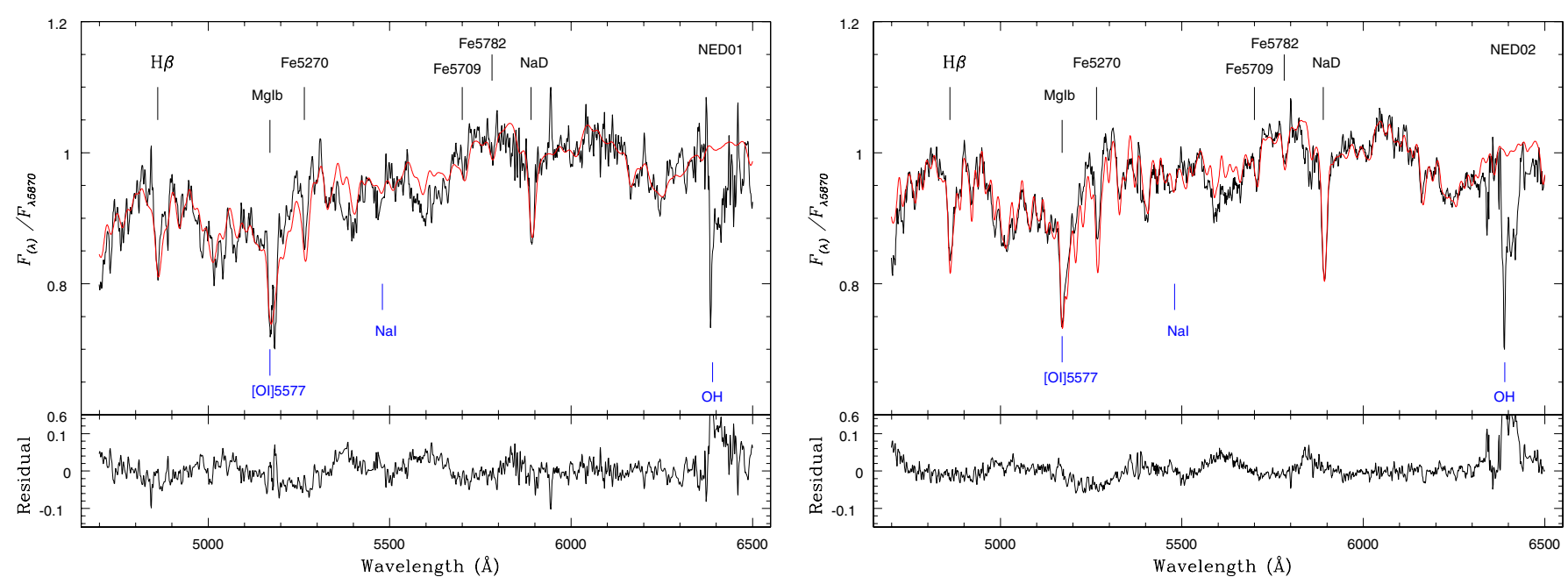

Fig. 4. Stellar population synthesis for NED01 and NED02. Observed spectra corrected for reddening (black line) and the synthesized spectrum (red line). The main absorption features have been identified and the most significant telluric lines and bands marked. The spectra are at rest frame.

Table 2. Stellar-population synthesis results (see text).

\begin{tabular}{|c|c|c|c|c|c|c|c|c|c|c|}
\hline Galaxy & $\begin{array}{c}x_{\mathrm{Y}} \\
\text { (per cent) }\end{array}$ & $\begin{array}{c}x_{\mathrm{I}} \\
\text { (per cent) }\end{array}$ & $\begin{array}{c}x_{\mathrm{O}} \\
\text { (per cent) }\end{array}$ & $\begin{array}{c}m_{\mathrm{Y}} \\
\text { (per cent) }\end{array}$ & $\begin{array}{c}m_{\mathrm{I}} \\
\text { (per cent) }\end{array}$ & $\begin{array}{c}m_{\mathrm{O}} \\
\text { (per cent) }\end{array}$ & $Z_{\star}^{1}$ & $\chi^{2}$ & $\begin{array}{l}\text { Adev } \\
\text { (mag) }\end{array}$ & $A_{v}$ \\
\hline NED01 & 0.0 & 0.0 & 100.0 & 0.0 & 0.0 & 100.0 & 0.03 & 2.1 & 1.57 & 0.23 \\
\hline NED01(old) & 0.0 & 0.0 & 100.0 & 0.0 & 0.0 & 100.0 & 0.02 & 2.2 & 1.56 & -0.04 \\
\hline NED02 & 13.0 & 0.0 & 87 & 0.1 & 0.0 & 99.9 & 0.04 & 2.0 & 1.05 & 0.27 \\
\hline NED02(old) & 0.0 & 0.0 & 100.0 & 0.0 & 0.0 & 100.0 & 0.03 & 2.2 & 1.12 & -0.05 \\
\hline
\end{tabular}

Notes. ${ }^{(1)}$ Abundance by mass with $Z_{\odot}=0.02$.

aspects related to the interacting process and to its effects on the properties of the individual galaxies and their evolution.

To investigate the star formation history of NED01 and NED02, we used the stellar population synthesis code STARLIGHT (Cid Fernandes et al. 2004, 2005; Asari et al. 2007). This code is extensively discussed in Cid Fernandes et al. (2004, 2005), and is built upon computational techniques originally developed for empirical population synthesis with additional ingredients from evolutionary synthesis models. This method was also used by Krabbe et al. (2011) and has been successful in describing the stellar population in interacting galaxies.

The code fits an observed spectrum $O_{\lambda}$ with a combination of $N_{\star}$ single stellar populations (SSPs) from the Bruzual $\&$ Charlot (2003) models. These models are based on a highresolution library of observed stellar spectra, which allows for detailed spectral evolution of the SSPs at a resolution of $3 \AA$ across the wavelength range of 3200-9500 $\AA$ with a wide range of metallicities. We used the Padova 1994 tracks as recommended by Bruzual \& Charlot (2003), with the initial mass function of Salpeter (1955) between 0.1 and $100 M_{\odot}$. Extinction is modeled by STARLIGHT as due to foreground dust, using the Large Magellanic Cloud average reddening law of Gordon et al. (2003) with $R_{V}=3.1$, and parametrized by the $V$-band extinction $A_{V}$. The SSPs used in this work cover 15 ages, $t=0.001$, $0.003,0.005,0.01,0.025,0.04,0.1,0.3,0.6,0.9,1.4,2.5,5,11$, and $13 \mathrm{Gyr}$, as well as three metallicities, $Z=0.2 Z_{\odot}, 1 Z_{\odot}$, and $2.5 Z_{\odot}$, added to the $N_{\star}=45 \mathrm{SSP}$ components.
The fitting was carried out using a simulated annealing plus Metropolis scheme, which searches for the minimum $\chi^{2}=\sum_{\lambda}\left[\left(O_{\lambda}-M_{\lambda}\right) w_{\lambda}\right]^{2}$ where $w_{\lambda}$ is the error in $O_{\lambda}$ and $M_{\lambda}$ the model spectrum. The model spectrum $M_{\odot}$ is obtained by the equation

$M_{\lambda}=M_{\lambda 0}\left[\sum_{j=1}^{N_{\star}} x_{j} b_{j}, \lambda r_{\lambda}\right] \otimes G\left(v_{\star}, \sigma_{\star}\right)$

where $b_{j}, \lambda r_{\lambda}$ is the reddened spectrum of the $j$ th SSP normalized at $\lambda_{0} ; r_{\lambda}=10^{0.4\left(A_{\lambda}-A_{\lambda_{0}}\right)}$ is the reddening term; $M_{\lambda 0}$ is the synthetic flux at the normalization wavelength; $\boldsymbol{x}$ is the population vector; $\otimes$ denotes the convolution operator; and $G\left(v_{\star}, \sigma_{\star}\right)$ is the Gaussian distribution used to model the line-of-sight stellar motions. It is centred at velocity $v_{\star}$ with dispersion $\sigma_{\star}$. Bad pixels and spurious features are masked out by fixing $w_{\lambda}=0$, such as the region around $\mathrm{Mg}_{1}$ that was affected by sky residuals. We keep the kinematical parameters fixed during the fit, assuming the values of the velocity dispersion obtained from the cross-correlation.

Prior to the modeling, the SSPs models were convolved to the same resolution of the observed spectra: the observed spectra were shifted to its rest frame, corrected for foreground Galactic reddening of $E(B-V)=0.053$ mag taken from Schlegel et al. (1998), and normalized to $\lambda 5870 \AA$. The observed spectra were resampled in steps of $\Delta \lambda=1 \AA$, the same as for the SSPs models. We synthesized the stellar population for the integrated spectrum (all galaxy) of NED01 and NED02. 
Figure 4 shows the observed spectra corrected for reddening, the model stellar population spectra, and the residual spectra for NED01 and NED02. The results of the synthesis are summarized in Table 2 for the integrated spectrum of each galaxy, stated as the perceptual contribution of each base element to the flux at $\lambda 5870 \AA$. Following the prescription of Cid Fernandes et al. (2005), we defined a condensed population vector by binning the stellar populations according to the flux contributions into young, $x_{\mathrm{Y}}\left(t \leq 5 \times 10^{7} \mathrm{yr}\right)$; intermediate-age, $x_{\mathrm{I}}\left(5 \times 10^{7}<\right.$ $\left.t \leq 2 \times 10^{9} \mathrm{yr}\right)$; and old, $x_{\mathrm{O}}\left(2 \times 10^{9}<t \leq 13 \times 10^{9} \mathrm{yr}\right)$ components. The same bins were used to represent the mass components of the population vector $\left(m_{\mathrm{Y}}, m_{\mathrm{I}}\right.$, and $\left.m_{\mathrm{O}}\right)$. The metallicity $(Z)$, one important parameter for characterizing the stellar population content, is weighted by the light fraction. The quality of the fitting result is measured by the parameters $\chi^{2}$ and adev. The latter gives the perceptual mean deviation $\left|O_{\lambda}-M_{\lambda}\right| / O_{\lambda}$ over all fitted pixels, where $O_{\lambda}$ and $M_{\lambda}$ are the observed and model spectra, respectively.

The results indicate that NED01 is completely dominated by an old stellar population of $2 \times 10^{9}<t \leq 13 \times 10^{9} \mathrm{yr}$. For NED02, a small fraction of about $13 \%$ of young stars with ages $\leq 5 \times 10^{7}$ yr contribute to the optical flux at $\lambda 5870 \AA$. As can be seen in Fig. 4, the synthetized spectra show small disagreement (on average $<5 \%$ ) with observed spectra in both galaxies, such as at about $15600 \AA$. Besides this, no emission lines were detected in the spectra of NED02, which weakens the conclusion about the small contribution of young star population from the synthesis for NED02. Concerning about it, we used only SSPs with old ages and different metallicities in the synthesis fitting. These results are shown in Table 2. The residuals found in this case almost have the same magnitude as the one found when SSPs with different ages are used. However, it resulted in very low extinction values of $A_{v}$. The error of $A_{v}$ estimations is on the order of $0.1 \mathrm{mag}$ (Cid Fernandes et al. 2013). According to Cid Fernandes et al. (2005), in the fitting the extinction $A_{v}$ is not constrained to be positive. Many reasons are pointed out by the authors for this choice, among them, that some objects may indeed require bluer SSP spectra than those in the base and that the observed light may contain a scattered component, which would induce a bluening of the spectra not taken into account by adopted pure exctinction law (for more details see in quoted article). Similar results were found for HRG 2304, a Solitaire galaxy candidate in an early stage of ring formation, and its companion AM 1646-795 (Wenderoth et al. 2011).

\section{Discussion}

In the FM 047-02 system, the NED01 galaxy is seen almost faceon and is clearly ringed, with two or three prominent smooth structures on the S and SE sides of the ring. No clearly prominent knots are visible in other regions of the ring, as detected in the Solitaire-candidate galaxies AM 2152-592, AM 2012-282, and AM 1434-783. As can be seen in Fig. 1, the object shows two clear signatures of tidal perturbations: one main external ring and an internal loop-ring-like structure extending to the SW.

NED01 resembles the collisional RE class of rings (empty rings) proposed by Theys \& Spiegel (1976). Recently, Mapelli \& Mayer (2012) have investigated the formation of RE galaxies from $N$-body simulations. The RE galaxies are characterized by a ring that is empty in its interior, and apparently lacking the nucleus. The RE objects happen to have high star formation rates, suggesting that the density wave associated with the propagating ring triggers the formation of stars. From off-center collision simulations, Mapelli \& Mayer (2012) demonstrate that the nucleus of the target galaxy is displaced from the dynamical center of the galaxy and placed within the ring. This result could explain the NED01 morphology.

In both FM 047-02 galaxies, along the entire slit, the spectra show features that are characteristic of early-type objects. No star-forming regions and no nuclear ionization sources were detected. The two galaxies form a tidally bound system with a radial velocity difference of almost $240 \mathrm{~km} \mathrm{~s}^{-1}$. The velocity dispersions $\sigma_{v}$ of 387 (NED01) and $285 \mathrm{~km} \mathrm{~s}^{-1}$ (NED02) were estimated by cross-correlation with $\mathrm{K}$ and $\mathrm{M}$ star templates, with the statistical error in the range of $10 \%-15 \%$ (XCS AO/RVS AO, also tested with $X C O R / S T S D A S$ ). We determined the dynamical masses for both galaxies based on the virial equation, assuming that both galaxies are at a distance of $322 \mathrm{Mpc}$. Using the Gemini pointing image and the Digitized Sky Survey one, we adopted the effective radii of 12 '. 7 and $11^{\prime \prime} .5$ and velocity dispersions of $387 \mathrm{~km} \mathrm{~s}^{-1}$ and $285 \mathrm{~km} \mathrm{~s}^{-1}$, respectively. The calculated mass of NED01 $\left(3.97 \times 10^{11} M_{\odot}\right)$ is approximately twice that of the mass of NED02 $\left(1.93 \times 10^{11} M_{\odot}\right)$. Besides this, there are no prominent field galaxies within 9 arcmin of FM 047-02, but there are several spherical objects that resemble dwarf galaxies within 2.5 arcmin and an elliptical-like object on the W of NED02 (with coordinates $\alpha=20^{\mathrm{h}} 26^{\mathrm{m}} 29^{\mathrm{s}} .22$ and $\delta=-72^{\circ} 35^{\prime} 55^{\prime \prime} .0,2000$ ). In this context, the galaxy pair FM 047-02 could be the main objects of a group of galaxies.

The observed radial-velocity distribution of NED01 shows some kinematically decoupled components, which are the products of the tidal interaction with NED02. These kinematic subsystems, displayed in Fig. 2, are unevenly distributed, including the very central region of this galaxy, of which center has a decoupled NE-section approaching us (on the side in the direction of NED02), and the almost rigid section including the very center between 0.'0 and 1'.25 approximately. The lower panel of Fig. 2 displays the almost "undisturbed" rotation curve in the interval $\pm 1^{\prime \prime}$ for NED01. This suggests that when the galaxy has a low degree of internal rotation, the tidal coupling should produce this U-type profile.

In this section we review the main kinematic structures for the FM 047-02 system based on the spectroscopy. When inspecting Figs. 2 and 3 with respect to both heliocentric velocity centers, the following is suggested: (a) the core of NED02 is counter-rotating; (b) the external region of NED02 is kinematically decoupled from its central region (while the NE section is approaching us, the SW section is receding from us); and (c) both sections of the NED01 ring are receding from us, along with the internal loop-ring. There are two phenomena inside the U-shaped-base of NED01: (1) the whole central region is displaced in the direction of the companion (NE direction), a phenomenon also seen in the aforementioned interacting pair HRG 2304; (2) the kinematical center is also displaced in the NE direction.

The spectroscopic results corroborate the suggestion that both galaxies are early-type objects. It is important to emphasize that the fitting of stellar population pointed out a small, but non-negligible, fraction of young stars along the radius of galaxy NED02 (about 10\%). On other hand, the galaxy NED01 is dominated by an old star population. No intermediate stellar populations have been identified (see Table 2). Similar results have also been found for the peculiar pRG HRG 2304 and its companion NED01 (Wenderoth et al. 2011), with a fraction of the young population along the radius of both galaxies (almost 9\%), but with some portion of intermediate stellar population. 
Studies of the fundamental plane and studies based on absorption line spectroscopy favor a "frosting" model in which early-type galaxies consist of an old base population with a small amount of younger stars (Trager et al. 2000; Gebhart et al. 2003; Schiavon 2007). In addition, data on early-type galaxies that exhibit strong ultraviolet excess, polycyclic aromatic hydrocarbon emission, and infrared excess are interpreted as a possible result of recent low-level star formation (Yi et al. 2005; Rich et al. 2005; Schawinski et al. 2007; Kaviraj et al. 2007; Temi et al. 2009; Young et al. 2009; Salim \& Rich 2010). Studies based on spatially resolved spectroscopy by Shapiro et al. (2010) and Kuntschner et al. (2010) have found that star formation in early-type galaxies happens exclusively in fastrotating objects and occurs in two different contexts: (a) objects with a widespread young stellar population associated with a high molecular gas content; and (b) objects with disk and/or ring morphology. The latter could be explained by rejuvenation in previously quiescent stellar systems (Shapiro et al. 2010; Kuntschner et al. 2010), and seems to be suitable to explaining both galaxies of FM 047-02.

The ring structure of NED01 was probably triggered by a wave of enhanced density moving outwards, resulting only in the redistribution of the old stellar population of this galaxy. This structure resembles the asymmetric distribution of the galaxy contents seen in the simulations from off-center collisions performed by Appleton \& Struck-Marcell (1987) and Mapelli \& Mayer (2012).

\section{Conclusions}

In this work, we report observational results of the pRG NED01 and its companion NED02, both members of FM 047-02. The galaxy NED01 was previously classified as a Solitaire-like galaxy by FAOA. Our work is based on spectroscopic observations in the optical. Below is a summary of our main results:

- The FM047-02 system is composed of the ring galaxy NED01 and the elliptical-like galaxy NED02, (2MASX J20262950-7236443 and 2MASX J202633967236083 , in NED, respectively). The projected separation between both galaxies' centers is almost $60 \mathrm{kpc}$. The velocity difference is close to $\Delta v=240\left(\mathrm{~km} \mathrm{~s}^{-1}\right)$, confirming that both galaxies are interacting close companions.

- Several spherical dwarf objects lie within 2.5 arcmin of FM 047-02. In the NW direction of NED02, an elliptical galaxy is close enough to be a field satellite, but there is no quoted redshift for this object in the literature. The galaxy pair FM 047-02 seems to be surrounded by a group of dwarf objects.

- The spectra of both galaxies resemble that of an early-type galaxy. No emission lines are detected in the observed sections of the NED01 ring, and no nuclear ionization sources were detected.

- Both observed distributions of the rotation profile show some kinematically decoupled components. These are the results of the tidal interaction between both objects. The discrete U-shaped rotation profile in NED01 $\left( \pm 5^{\prime \prime}\right)$ is thus direct observational evidence of tidal coupling, hence a direct observational signature of tidal friction in action with NED02.

- Both galaxies are dominated by old stellar populations with ages between $2 \times 10^{9}<t \leq 13 \times 10^{9}$ yr. About $13 \%$ of young stars are estimated to contribute to the optical flux for NED02.
- The morphology, kinematical properties, the early-type characteristics of the central region, the smoothness of the ring around NED01, and its off-center nucleus all characterize it as a true archetype of a Solitaire ring galaxy in an advanced stage of ring formation.

NED01 is a pRG where signatures of tidal perturbations are clearly visible. One ring and an internal ring-like structure are visible in Fig. 1. This remarkable system may have been generated by an off-center collision, as demostrated by $N$-body simulations by Mapelli \& Mayer (2012), the disk of NED01 having given rise to the ring. A probably early stage of this system could be represented by the galaxy pair IC 5364 (AM 2353-291), a clearly interacting system with a velocity difference of $\Delta V=22 \mathrm{~km} \mathrm{~s}^{-1}$, and when in an advanced stage of dynamical evolution, by AM 2145-543, which is probably the result of a off-center impact. We intend to conduct an exploratory study of FM 047-02 by employing numerical $N$-body/hydrodynamical simulation to reconstruct its history and to predict the evolution of this tidal interaction.

Acknowledgements. This work was partially supported by the Ministerio da Ciência, Tecnologia e Inovação (MCTI), Laboratório Nacional de Astrofísica (LNA), and Universidade do Vale do Paraíba - UNIVAP. A. C. Krabbe thanks the support of FAPESP, process 2010/01490-3. The paper is based on observations obtained at the Gemini Observatory, which is operated by the Association of Universities for Research in Astronomy, Inc., under a cooperative agreement with the NSF on behalf of the Gemini partnership: the National Science Foundation (US), the Science and Technology Facilities Council (UK), the National Research Council (Canada), CONICYT (Chile), the Australian Research Council (Australia), Ministrio da Ciłncia e Tecnologia (Brazil) and Ministerio de Ciencia, Tecnologa e Innovacin Productiva (Argentina). The observations were performed under the identification number GS-2007A-DD-06. This publication makes use of data products from the Two Micron All Sky Survey, which is a joint project of the University of Massachusetts and the Infrared Processing and Analysis Center/California Institute of Technology, funded by the National Aeronautics and Space Administration and the National Science Foundation. This research made use of the NASA/ IPAC Infrared Science Archive, which is operated by the Jet Propulsion Laboratory, California Institute of Technology, under contract with the National Aeronautics and Space Administration.

\section{References}

Appleton, P. N., \& Struck-Marcell, C. 1987, ApJ, 318, 103

Appleton, P. N., \& Struck-Marcell, C. 1996, in Fundamentals of Cosmic Physics, 16,111

Arp, H. C., \& Madore, B. F. 1986, Catalogue of Southern Peculiar Galaxies and Associations, I, Positions and Descriptions, Clarke-Irwin, Toronto

Asari, N. V., Cid Fernandes, R., Stasińska, G., et al. 2007, MNRAS, 381, 263

Astier, P., Guy, J., Regnault, N., et al. 2006, A\&A, 447, 31

Balcells, M., \& González, A. C. 1998, ApJ, 505, L109

Balcells, M., Borne, K. D., \& Hoessel, J. G. 1989, ApJ, 336, 655

Baldwin, J. A., \& Stone, R. P. S. 1984, MNRAS, 206, 241

Bender, R., Paquet, A., \& Nieto, J.-L. 1991, A\&A, 246, 349

Borne, K. D. 1988, ApJ, 330, 38

Borne, K. D. 1990, in Dynamics and Interactions of Galaxies, ed. R. Wielen (Berlin: Springer), 196

Borne, K. D., \& Hoessel, J. G. 1985, BAAS, 17, 601

Borne, K. D., \& Hoessel, J. G. 1988, ApJ, 330, 51

Borne, K. D., Balcells, M., Hoessel, J. G., \& McMaster, M. 1994, ApJ, 435, 79

Buta, R. 1995, ApJS, 96, 39

Bruzual, G., \& Charlot, S. 2003, MNRAS, 344, 1000

Cid Fernandes, R., Gu, Q., Melnick, J., et al. 2004, MNRAS, 355, 273

Cid Fernandes, R., Mateus, A., Sodré, L., Stasińska, G., \& Gomes, J. M. 2005, MNRAS, 358, 363

Cid Fernandes, R., González Delgado, R., García Benito, R., et al. 2013, A\&A, in press, DOI: 10.1051/0004-6361/201321692

De Rijcke, S., Dejonghe, H., Zeilinger, W. W., \& Hau, G. K. T. 2004, A\&A, 426, 53

Faúndez-Abans, M., \& de Oliveira-Abans, M. 1998, A\&AS, 129, 357 (FAOA)

Freedman, W. L., Madore, B. F., Gibson, B. K., et al. 2001, ApJ, 553, 47

Gebhardt, K., Faber, S. M., Koo, D. C., et al. 2003, ApJ, 597, 239 
Gordon, K. D., Clayton, G. C., Misselt, K. A., Landolt, A. U., \& Wolff, M. J. 2003, ApJ, 594, 279

Hamuy, M., Walker, A. R., Suntzeff, N. B., et al. 1992, PASP, 104, 533

Hamuy, M., Suntzeff, N. B., Heathcote, S. R., et al. 1994, PASP, 106, 566

Hau, G. K. T., \& Thomson, R. C. 1994, MNRAS, 270, L23

Hook, I. M., Jorgensen, I., Allington-Smith, J. R., et al. 2004, PASP, 166, 425

Jones, D. H., Read, M. A., Saunders, W., et al. 2009, MNRAS, 399, 683

Kaviraj, S., Schawinski, K., Devriendt, J. E. G., et al. 2007, ApJS, 173, 619

Krabbe, A. C., Pastoriza, M. G., Winge, C., et al. 2011, MNRAS, 416, 38

Kuntschner, H., Emsellem, E., Bacon, R., et al. 2010, MNRAS, 408, 97

Lang, K. R. 1999, Astrophysical Formulae, II, Space, Time, Matter and Cosmology, 3rd edn. (Springer-Verlag)

Madejsky, R. 1991, A\&A, 247, 348

Madejsky, R., Bender, R., \& Möllenhoff, C. 1991, A\&A, 242, 58

Madore, B. F., Nelson, E., \& Petrillo, K. 2009, ApJS, 181, 572

Mapelli, M., \& Mayer, L. 2012, MNRAS, 420, 1158

Rich, R. M., Salim, S., Brinchmann, J., et al. 2005, ApJ, 619, L107
Salim, S., \& Rich, R. M. 2010, ApJ, 714, L290

Salpeter, E. E. 1955, ApJ, 121, 161

Shapiro, K. L., Falcon-Barroso, J., van de Ven, G., et al. 2010, MNRAS, 402, 2140

Schawinski, K., Kaviraj, S., Khochfar, S., et al. 2007, ApJS, 173, 512

Schiavon, R. P. 2007, ApJS, 171, 146

Schlegel, D. J., Finkbeiner, D. P., \& Davis, M. 1998, ApJ, 500, 525

Spergel, D. N., Verde, L., Peiris, H. V., et al. 2003, ApJS, 148, 175

Temi, P., Brighenti, F., \& Mathews, W. G. 2009, ApJ, 695, 1

Theys, J. C., \& Spiegel, E. A. 1976, ApJ, 208, 650

Tranger, S. C., Faber, S. M., Worthey, G., \& González, J. J. 2000, AJ, 119, 1645

van Zee, L., Skillman, E. D., \& Haynes, M. P. 2004, AJ, 128, 121

Wenderoth, E., Faúndez-Abans, M., Krabbe, A. C., de Oliveira-Abans, M., \& Cuevas, H. 2011, A\&A, 529, A157

Yi, S. K., Yoon, S. J., Kaviraj, S., et al. 2005, ApJ, 619, L111

Young, L. M., Bendo, G. J., \& Lucero, D. M. 2009, AJ, 137, 3053 